\title{
The observability of gamma-ray spectral features from Kaluza-Klein dark matter annihilation
}

\author{
Satoshi Tsuchida* \\ Affiliation: Department of Physical Sciences, Ritsumeikan University \\ E-mail: re005076eed.ritsumei.ac.jp

\section{Masaki Mori} \\ Affiliation: Department of Physical Sciences, Ritsumeikan University \\ E-mail: morimefc.ritsumei.ac.jp
}

\begin{abstract}
The lightest Kaluza-Klein particle (LKP), which appears in the theory of universal extra dimensions, is one of the good candidates for cold dark matter. We assume the LKP mass is in the range from $500 \mathrm{GeV}$ to $1000 \mathrm{GeV}$, and focus on the LKP annihilation modes which contain gammarays as final products. The gamma-ray spectrum from LKP annihilation has a characteristic peak structure near the LKP mass ( "line") from two-body decays and continuum emission extending to lower energies. Gamma rays do not lose energy during propagation after production near the galactic center where dark matter concentration is expected, so we can treat it easier than electron. We investigate the detectability of the peak structure by considering energy resolution of near-future detector, and calculate the expected count spectrum of the gamma-ray signal. The observed gamma-ray spectrum will show the peak clearly, if the LKP mass is heavier. In contrast, if the LKP mass is light, constraint for the boost factor should come from the abundant continuum emission. Detecting such peak structure would be conclusive evidence that dark matter is made of LKP.
\end{abstract}

The 34th International Cosmic Ray Conference,

30 July- 6 August, 2015

The Hague, The Netherlands

\footnotetext{
* Speaker.
} 


\section{Introduction}

The dark matter problem, which was suggested by F. Zwicky [四], is one of the most important mysteries in cosmology and particle physics [[]]. Various observational data show some indirect evidence supporting the existence of dark matter. One of the methods to consider what makes dark matter is to suppose that particles predicted by new theories, which have not yet been detected, are candidates for dark matter [B]. One feasible candidate is the weakly interacting massive particle (WIMP). WIMPs are good candidates for cold dark matter (CDM), where cold implies the matter has a non-relativistic velocity at the freeze-out time in the early Universe.

The theory of universal extra dimensions (UED) is a popular theory beyond the standard model [四], where universal means that all fields of the standard model can propagate into extra dimensions. New particles predicted by this theory are called Kaluza-Klein (KK) particles. Here, we consider the theory of UED containing only one extra dimension. The extra dimension is compactified with radius $R$. At tree level, the KK particle mass is given by [[] ]

$$
m^{(n)}=\sqrt{\left(\frac{n}{R}\right)^{2}+m_{\mathrm{EW}}^{2}}
$$

where $n$ is a mode of the KK tower, and $m_{\mathrm{EW}}$ is a zero mode mass of an electroweak particle.

We assume that the lightest KK particle (LKP) is a feasible candidate for dark matter, and we denote it as $B^{(1)}$. $B^{(1)}$ is the first KK mode of the hypercharge gauge boson. Dark matter should be electrically neutral and almost stable. Hence, the LKP either does not interact with the standard model particles or only weakly interacts with them. In addition, LKP should have a very small decay rate to survive for a cosmological time. In order for LKP to have a significant relic density, the LKP mass $m_{B^{(1)}}$ should be in the range $500 \mathrm{GeV} \lesssim m_{B^{(1)}} \lesssim 1 \mathrm{TeV}$. In this paper, we assume the $m_{B^{(1)}}$ is $800 \mathrm{GeV}$ firstly, then we consider the change of result in the mass range of $500 \mathrm{GeV}$ to $1000 \mathrm{GeV}$.

There are many LKP annihilation modes which contain gamma-rays as final products. These include gamma-ray "lines" from two-body decays, and "continuum" emission from decay or fragmentation of secondaries. The cross section for $B^{(1)}$ pair annihilation has been calculated [目], and we assume the mass splitting is $5 \%$ at the first KK level. In addition, branching ratios into these

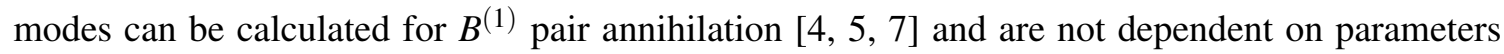
other than $m_{B^{(1)}}$. This paper considers three patterns for the continuum: $B^{(1)}$ pairs annihilate into (i) quark pairs, (ii) lepton pairs which cascade or produce gamma-rays, or (iii) two leptons and one photon $\left(l^{+} l^{-} \gamma\right)$. The gamma-ray spectra of the continuum component for $m_{B^{(1)}}=800 \mathrm{GeV}$ are given in Ref. [四].

When $B^{(1)}$ pairs annihilate into photon pairs, they appear as a "line" at $m_{B^{(1)}}$ in the gammaray spectrum. This is the most prominent signal of KK dark matter, while in some theories line modes are loop-suppressed and thus usually subdominant (see, e.g. []] ). This study focuses on the detectability of this "line" structure by near-future detector taking account of their finite energy resolution.

The distribution of dark matter is expected to be non-uniform in the Universe, and to be concentrated in massive astronomical bodies due to gravity. Then, we consider the "boost factor" which indicates the relative concentration of the dark matter in astronomical bodies compared 
with some benchmark distributions, such as Navarro-Frenk-White (NFW) [Q]. The boost factor is affected by $\langle\sigma v\rangle$ and $\rho^{2}(l)$, and is defined by

$$
B_{\mathrm{tot}}=B_{\rho} \times B_{\sigma v}=\left(\frac{\left\langle\rho^{2}(l)\right\rangle_{\Delta V}}{\left\langle\rho_{0}^{2}(l)\right\rangle_{\Delta V}}\right)\left(\frac{\langle\sigma v\rangle_{v \simeq v_{\text {disp }}}}{\langle\sigma v\rangle_{v \simeq v_{\mathrm{F}}}}\right)_{\Delta V},
$$

where $v_{\text {disp }}$ is the velocity dispersion, $v_{\mathrm{F}}$ is the typical velocity at freeze-out, the volume $\Delta V$ is a diffusion scale, and $\rho_{0}(l)$ is a typical dark matter density profile. $B_{\rho}$ could be as high as 1000 when taking account of the expected effects of adiabatic compression [प]]. In the case of gamma-ray flux from LKP annihilation, the particle physics factor is almost fixed for a given model, so the boost factor mostly depends on the astrophysical contribution.

Some constraints from observations on the KK dark matter models have been reported. The Fermi-LAT team searched for gamma-ray emission from dwarf spheroidal galaxies around the Milky Way galaxy and set constraints on dark matter models with non-detection results [ $\square]$ ]. The HESS array of imaging air Cherenkov telescopes observed the Sagittarius dwarf spheroidal galaxy in the sub-TeV energy region and derived lower limits on the $m_{B^{(1)}}$ of $500 \mathrm{GeV}$ [ए2]. These results put constraints on $\langle\sigma v\rangle$ of dark matter halo KK particles. The current limits allow the maximum value of boost factors in the range of $2-60\left(m_{B^{(1)}}=200 \mathrm{GeV}\right)$ to $600-1.5 \times 10^{4}(1000 \mathrm{GeV})$ by Fermi-LAT [山]], 0.8 - $30(400 \mathrm{GeV})$ to 5 - $160(1000 \mathrm{GeV})$ by HESS [ए3]].

In the following, we focus on gamma-ray observation with near-future missions, such as the Calorimetric Electron Telescope (CALET) [पय]]. CALET is a fine resolution calorimeter for cosmic-ray observation to be installed on the International Space Station. CALET will detect gamma-rays in the energy range of $4 \mathrm{GeV}$ to $1 \mathrm{TeV}$ with about $1000 \mathrm{~cm}^{2}$ effective area and a few percent energy resolution, suitable for gamma-ray line detection [피].

In this paper, we analyze the gamma-ray spectral features from $B^{(1)}$ pair annihilation taking account of the finite energy resolution of gamma-ray detector and discuss the observability of the "line" at the $m_{B^{(1)}}$. We then give possible constraints on the boost factor by near-future detector. The essential contents of this paper has been submitted for publication elsewhere.

\section{The effect of energy resolution}

The gamma-ray flux $d \Phi_{\gamma}(\Delta \Omega) / d E_{\gamma}$ reaching a detector can be expressed as [[]]

$$
E_{\gamma}^{2} \frac{d \Phi_{\gamma}(\Delta \Omega)}{d E_{\gamma}} \simeq \text { Const } \times B_{\text {tot }} \times x^{2} \frac{d N_{\gamma}}{d x}
$$

where $\Delta \Omega$ is the angular acceptance of the detector,

$$
\text { Const } \simeq 3.5 \times 10^{-8}\left(\frac{\langle\sigma v\rangle}{3 \times 10^{-26} \mathrm{~cm}^{3} \mathrm{~s}^{-1}}\right)\left(\frac{0.8 \mathrm{TeV}}{m_{B^{(1)}}}\right)\left\langle J_{\mathrm{GC}}\right\rangle_{\Delta \Omega} \Delta \Omega
$$

and $\left\langle J_{\mathrm{GC}}\right\rangle_{\Delta \Omega}$ is a dimensionless line-of-sight integral averaged over a solid angle $\Delta \Omega$. We set $\langle\sigma v\rangle=1.3 \times 10^{-4} \mathrm{pb}$, and we assume a $5 \%$ mass splitting at the first KK level following Ref. [可]. The boost factor is calculated based on this cross section value. If we assume an NFW profile, $\left\langle J_{\mathrm{GC}}\right\rangle_{\Delta \Omega} \Delta \Omega$ equals to 0.39 for a $\Delta \Omega=10^{-4}$ [ए]], which is a reasonable value both for the angular resolution of CALET $\left(0.2-0.3^{\circ}\right)$ [ए5] and the observed localization of the galactic center 


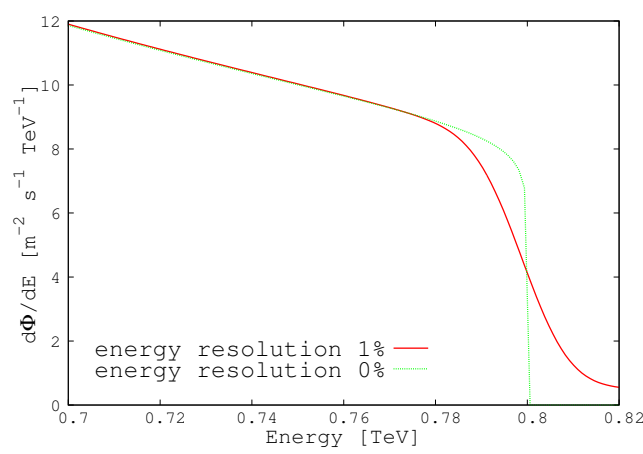

Figure 1: (Color online) Gamma-ray spectrum of the continuum taking account of the energy resolution assuming $m_{B^{(1)}}=800 \mathrm{GeV}$. The solid line assumes an energy resolution of $1 \%$ with a Gaussian distribution, and the dotted line does not include the effect of energy resolution. The assumed boost factor is 100 .

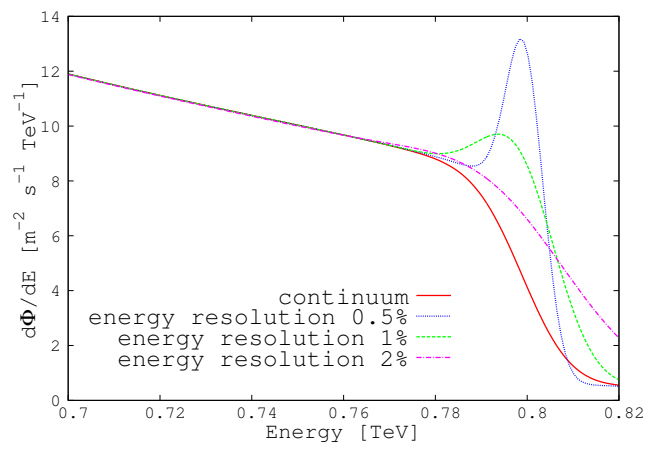

Figure 2: (Color online) Gamma-ray spectra of continuum plus line diffused by the energy resolution assuming $m_{B^{(1)}}=800 \mathrm{GeV}$. The solid line shows the continuum component only, assuming the energy resolution of $1 \%$, while the dotted, dashed, and dot-dashed lines show the continuum plus line components assuming energy resolution values of $0.5 \%, 1.0 \%$, and $2 \%$, respectively. The assumed boost factor is 100 .

source observed by Fermi-LAT [प]]. In this case $d N_{\gamma} / d x$ includes both the continuum and line components.

Now, we discuss the effect of energy resolution of detectors. If the measured energy dispersion for mono-energetic gamma-rays behaves as a Gaussian distribution and the energy resolution of the detector is $1 \%$, the measured gamma-ray spectrum is blurred as shown in Fig.W for the "continuum" component. Here we draw the curve assuming the following equation

$$
g(E) \propto \int f\left(E^{\prime}\right) \times \exp \left[-\frac{\left(E-E^{\prime}\right)^{2}}{2 \sigma_{E}^{2}}\right] d E^{\prime},
$$

where $f\left(E^{\prime}\right)$ corresponds to a spectrum of the total continuum component, and $\sigma_{E}$ is the energy resolution.

Next we analyze how the "line" from the $B^{(1)}$ pair annihilation into photon pairs looks like above the "continuum". In Fig.】, the solid line shows the continuum component only with an energy resolution of $1 \%$, and the patterned lines show "line" plus "continuum" spectra for different energy resolutions: the dotted line, dashed line, and dot-dashed line show the spectra when the energy resolution is $1 \%, 0.5 \%, 2 \%$ with the Gaussian distribution, respectively, assuming the boost factor $B_{\text {tot }}=100$. We also point out that the peak energies of the expected spectra $\left(E_{\text {peak }}\right)$ are $0.2 \%$, $0.8 \%$, and $1.4 \%$ smaller than $m_{B^{(1)}}$, for $0.5 \%, 1 \%$, and $2 \%$ energy resolution, respectively.

We can transform the spectra into counts to be observed by gamma-ray detectors. When analyzing observational data, the energy bin width must be specified. Bin widths of twice as much as $0.5 \%, 1 \%$ and $2 \%$ of $m_{B^{(1)}}$ (about one standard deviation of energy reconstruction) was used in order to match the each energy resolution. The resulting histograms are shown in Fig. [3], where plots of the three cases corresponding to energy resolutions of $0.5 \%, 1 \%$ and $2 \%$ are shown. The figure shows that if the energy resolution of the detector becomes $2 \%$ or worse, the characteristic peak indicating the $m_{B^{(1)}}$ will be diffused, making it hard to resolve into the line and continuum 


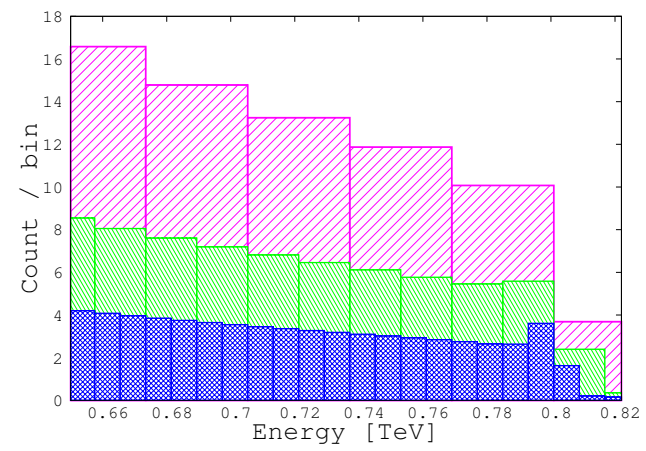

Figure 3: (Color online) Expected count spectra near the peak assuming energy resolutions of $0.5 \%$, $1 \%$ and $2 \%$ assuming $m_{B^{(1)}}=800 \mathrm{GeV}$. The bin widths of histograms are twice as much as $0.5 \%$, $1 \%$ and $2 \%$ of the $m_{B^{(1)}}$, respectively. The assumed boost factor is 100 .

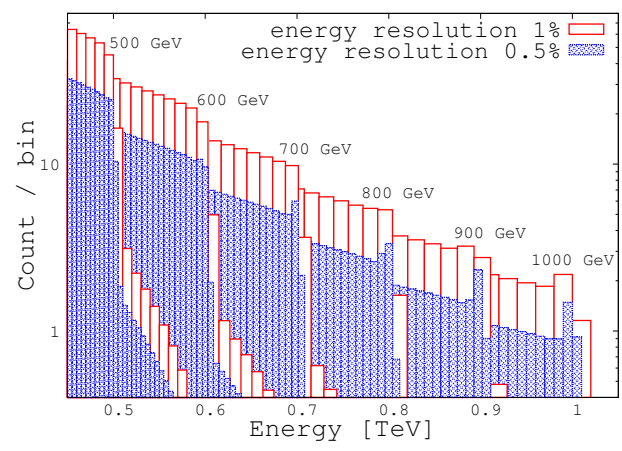

Figure 4: (Color online) Expected count spectra, assuming energy resolutions of $0.5 \%$ and $1 \%$. The data spaces are twice as much as $0.5 \%$ and $1 \%$ of the $m_{B^{(1)}}$. The assumed boost factor is 100 .

components. Here, we do not consider for the systematic error, because the shape of spectral features will be not changed. The energy resolution for gamma-ray detectors should be better than $2 \%$, in order to "resolve the line" without the need for detailed analyses.

Thus far, we have taken the LKP mass to be $m_{B^{(1)}}=800 \mathrm{GeV}$, and calculated count spectrum for its mass. Now, we vary the mass from $500 \mathrm{GeV}$ to $1000 \mathrm{GeV}$ in $100 \mathrm{GeV}$ intervals, and calculate the count spectrum for each mass. The results are shown in Fig.⿴囗十, which shows that the characteristic peak structure is visually clearer when $m_{B^{(1)}}$ is heavier. That is, the line component becomes relatively larger since the continuum component decreases for heavier $m_{B^{(1)}}$.

To investigate this tendency quantitatively, we consider the equivalent width for the line. The equivalent width $W$ is defined as

$$
W=\sum_{i} \frac{F_{i}^{c}-F_{i}^{l}}{F_{i}^{c}} \Delta E
$$

where $F_{i}^{c}, F_{i}^{l}$ are the counts of the continuum component and the line component of the $i$-th energy bin, respectively, and $\Delta E$ is the energy bin width which is set to $0.1 \%$ of each $m_{B^{(1)}}$. The summation runs from the lower to the upper energy limit of the observed line. This range corresponds to $\pm 5 \sigma_{E}$ for each $m_{B^{(1)}}$. The result is shown in Fig.[1 as a function of $m_{B^{(1)}}$. In this figure, we can see that the equivalent width increases as $m_{B^{(1)}}$ becomes heavier, which implies characteristic peak structure is clearer for heavier $m_{B^{(1)}}$.

\section{Discussion}

We now discuss the observability of the LKP signal in near-future detector, taking account of the observed background flux. That is, we give estimates for the accessible range of the boost factor when the observed counts are significantly different from the background flux. Here, we assume the gamma-ray flux from HESS J1745-290 located near the center of the Galaxy is the source of 


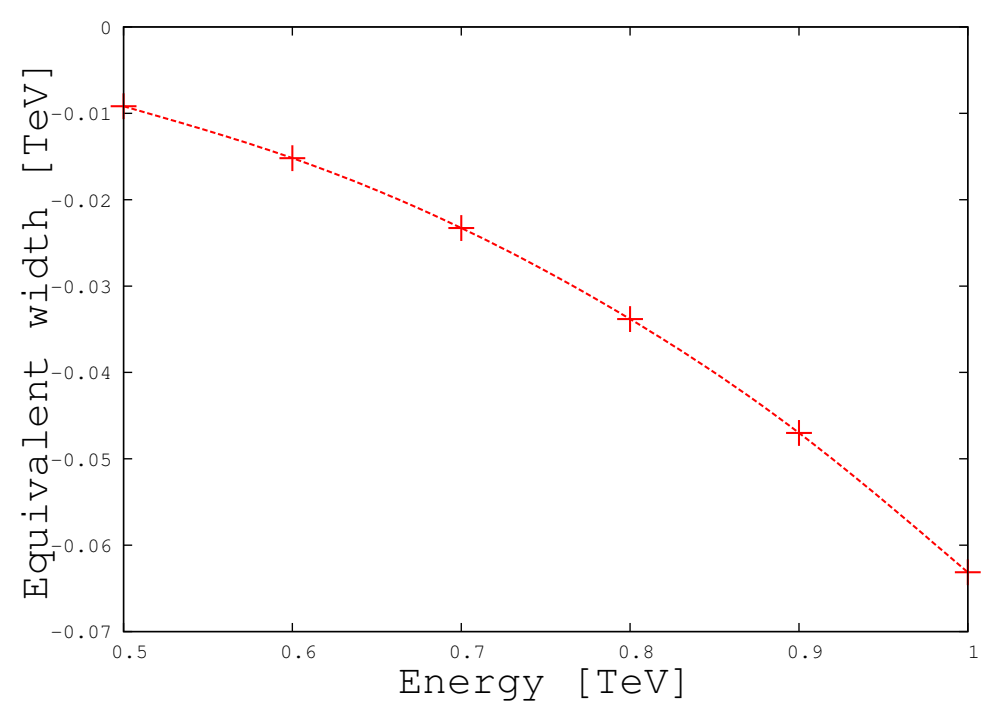

Figure 5: (Color online) Equivalent width as a function of $m_{B^{(1)}}$, assuming an energy resolution of $1 \%$. The dashed curve is drawn to guide to the eyes.

the background. Its flux is given by [[13]

$$
\begin{aligned}
\frac{d \Phi}{d E}= & (2.55 \pm 0.06 \pm 0.40)\left(\frac{E}{\mathrm{TeV}}\right)^{-2.10 \pm 0.04 \pm 0.10} \\
& \times \exp \left[-\frac{E}{(15.7 \pm 3.4 \pm 2.5) \mathrm{TeV}}\right] \times 10^{-8} \mathrm{TeV}^{-1} \mathrm{~m}^{-2} \mathrm{~s}^{-1} .
\end{aligned}
$$

Note that with the energy resolution of HESS (15\%), the LKP "line" signal is broadened and hard to detect.

To investigate the detectability quantitatively, we employ a $\chi$-squared test method. We hypothesize that the observed counts are not significantly different from the background. Thus, if the test indicates we should reject the hypothesis, this implies the observed counts are significantly different from the background. In this case, the excess counts should be regarded as LKP signals.

First, we define $\chi^{2}$ as

$$
\chi^{2}=\sum^{N} \frac{([\text { count }+ \text { background }]-\text { background })^{2}}{\text { background }},
$$

where $N$ is the number of energy bins, corresponding to degrees of freedom for the $\chi$-squared test. We then specify the energy range, as for example,

$$
\text { Energy range }=[100 \mathrm{GeV}, 1 \mathrm{TeV}],
$$

with bin width of $0.8 \mathrm{GeV}\left(=0.1 \%\right.$ for $\left.m_{B^{(1)}}=800 \mathrm{GeV}\right)$. Thus, $N$ is about 1000 in this case. The upper bound of the energy range under analysis is fixed as $m_{B^{(1)}}+3 \sigma_{E}$ to allow finite energy resolution. Hence, if we use this energy bin only, the degree of freedom is one $(N=1)$. Then, we vary the lower bound of the energy range to lower energies. Thus, $N$ gradually increases as we 


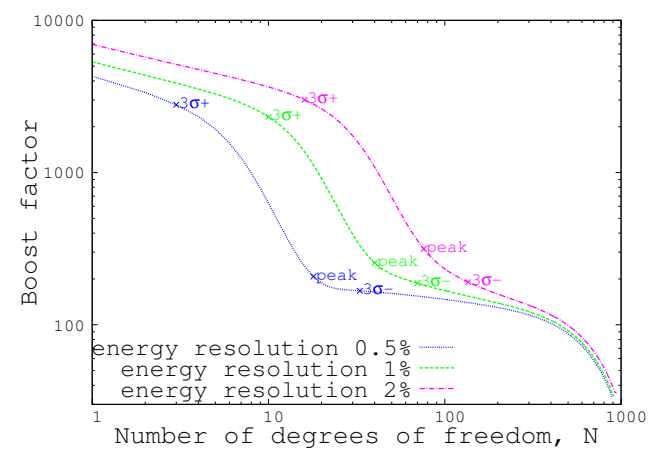

Figure 6: (Color online) Expected limits on the boost factor for the $m_{B^{(1)}}=800 \mathrm{GeV}$ as a function of the number of degrees of freedom of the observed energy range. The dotted, dashed and dot-dashed lines show the boost factor when $\chi^{2}$ values are bigger than critical values for energy resolution $0.5 \%$, $1.0 \%$, and $2 \%$ respectively.

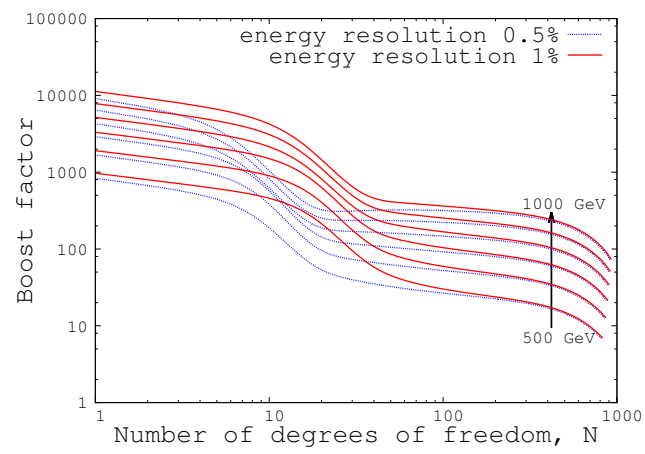

Figure 7: (Color online) Comparison of the expected limits on the boost factors with $1 \%$ and $0.5 \%$ energy resolution. The individual lines refer to the scenarios with masses set from $500 \mathrm{GeV}$ to 1000 $\mathrm{GeV}$ in $100 \mathrm{GeV}$ intervals.

expand the energy range to lower energies. For example, $N$ at the peak for $1 \%$ energy resolution is defined as: $N\left[E_{\text {peak }}, m_{B^{(1)}}+3 \sigma_{E}\right]=40$. We investigate the value of boost factor when $\chi^{2}$ is bigger than some critical value for each $N$. The relation between $N$ and the upper bound of the boost factor is shown in Fig.6, where the "peak" on each line corresponds to the value when $N$ equals to $N\left[E_{\text {peak }}, m_{B^{(1)}}+3 \sigma_{E}\right]$. Then, $3 \sigma^{ \pm}$are the energy range limits within $3 \sigma$ from the peak. Thus, they are given as: $N$ at $3 \sigma^{ \pm}=N\left[E_{\text {peak }} \pm 3 \sigma_{E}, m_{B^{(1)}} \pm 3 \sigma_{E}\right]$. One can see from Fig.6 that the limit on the boost factor does not change rapidly when we include energy bins well below the peak. An accessible boost factor would be smaller than 250 when $N$ is in the range 30-100. These values of $N$ correspond to being near the peak energy for annihilation of LKP.

We apply a similar analysis for other LKP masses, and compare the results for $1 \%$ energy resolution with $0.5 \%$ energy resolution, as shown in Fig.Ш. The results shown in this figure indicate that the constraint for the boost factor is tighter for lighter $m_{B^{(1)}}$. In the most restricted case, the upper limit of the boost factor will be about 10. In addition, because the number of events at the peak increases with better energy resolution, the resulting constraint becomes tighter. Thus our analysis indicates how future high-energy-resolution observation improves the limits on the boost factor, or increases the chance to detect the signal.

\section{Conclusion}

Energy resolution plays a key role in detecting the line structure of the gamma-ray spectral features expected from annihilation of LKP dark matter as predicted by UED theories. This paper investigated the effects of energy resolution of gamma-ray detector and calculated the expected count spectrum. The predicted gamma-ray spectrum is the sum of the continuum and the line corresponding to $m_{B^{(1)}}$, but this characteristic structure is diluted when we take account of the finite energy resolution of detectors as shown in Fig.W multiplied by observation time) of near-future detectors, count statistics will be the final limiting 
factor. The characteristic peak indicating the $m_{B^{(1)}}$ would be diffused if the energy resolution is $2 \%$ or worse. However, with qualitative statistical analysis, we may be able to detect a peak statistically by subtracting a background from the observed spectrum. In addition, if $m_{B^{(1)}}$ is heavy, the observed gamma-ray spectrum will show the characteristic peak clearly because the continuum component decreases relative to the line component.

This paper also estimated the accessible range of the boost factor using a $\chi$-squared test assuming the HESS J1745-290 spectrum as a background. If the observed energy range for gammarays extends to lower energies, the accessible range of the boost factor will be lowered since more continuum events will be detected. Assuming the detector having effective area of $1000 \mathrm{~cm}^{2}$, if the signal is not detected in one-year observation, the upper limit of the boost factor is about 250 for $m_{B^{(1)}}=800 \mathrm{GeV}$ if only taking data near the peak into account. Furthermore, if $m_{B^{(1)}}$ is light or the energy resolution of the detector is good (say the order of $0.5 \%$ ), we may tightly constrain on the boost factor (about 10 for $m_{B^{(1)}}=500 \mathrm{GeV}$ ).

If the gamma-ray line feature is observed in the future, we may identify LKP dark matter, which will provide strong evidence for the existence of extra dimensions.

\section{References}

[1] F. Zwicky, Helv. Phys. Acta. 6, 110 (1933).

[2] For a review, See, e.g., G. Bertone, D. Hooper, and J. Silk, Phys. Rep. 405, 279 (2005).

[3] L. Bergström, New J. Phys. 11, 105006 (2009).

[4] H. C. Cheng, K. T. Matchev, and M. Schmaltz, Phys. Rev. D 66, 036005 (2002).

[5] L. Bergström, T. Bringmann, M. Eriksson, and M. Gustafsson, Phys. Rev. Lett. 94, 131301 (2005).

[6] L. Bergström, T. Bringmann, M. Eriksson, and M. Gustafsson, J. Cosmol. Astropart. Phys. 04, 004 (2005).

[7] G. Servant and T.M.P. Tait, Nucl. Phys. B 650, 391 (2003).

[8] T. Bringmann and C. Weniger Physics of Dark Universe 1, 194 (2012).

[9] J. F. Navarro, C.S. Frenk, and S. D. White, Astrophys. J. 462, 563 (1996).

[10] F. Prada, A. Klypin, J. Flix, M. Martinez, and E. Simonneau, Phys. Rev. Lett. 93, 241301 (2004).

[11] A. A. Abdo et al. (Fermi-LAT collaboration), Astrophys. J. 712, 147 (2010).

[12] F. Aharonian et al., Astropart. Phys. 29, 55 (2008); Erratum Astropart. Phys. 33, 274 (2010)

[13] F. Ahanronian et al., Astron. Astrophys. 503, 817 (2009).

[14] S. Torii, Nucl. Instrum. Methods. A 630, 55 (2011).

[15] M. Mori et al., Proc. 33rd ICRC (Rio de Janeiro), Paper 0248 (2013).

[16] A. Cesarini, F. Fucito, A. Lionetto, A. Morselli, and P. Ullio, Astropart. Phys. 21, 267 (2004).

[17] M. Chernyakova, D. Malyshev, F. A. Aharonian, R. M. Crocker, and D. I. Jones, Astrophys. J. 726, 60 (2011). 